\title{
Controle postural e sensibilidade plantar em jovens e idosos
}

\section{Postural control and plantar sensitivity in young and elderly persons}

Como citar este artigo: ALFIERI, Fábio Marcon; VIEIRA, C. F.; VARCAS E SILVA, NCO. Controle postural e sensibilidade plantar em jovens e idosos. Revista Saúde (Sta. Maria). 2019; 45 (2).

\section{Autor correspondente:}

Nome: Fábio Marcon Alfieri

E-mail: fabio.alfieri@unasp.edu.br

Telefone: (1 1 ) 2128-6941

Formação Profissional: Doutor

em Ciências Médicas pela

Universidade de São Paulo, São

Paulo, SP, Brasil.

Filiação Institucional: Centro Universitário Adventista

Endereço para correspondência:

Estrada de Itapecerica n’: 5859

Bairro: Jardim IAE

Cidade: São Paulo Estado: São

Paulo CEP: 05858-00 1

Data de Submissão:

08/06/20 18

Data de aceite:

04/08/2019

Conflito de Interesse: Não há conflito de interesse

\section{(cc) $\mathrm{BY}-\mathrm{NC}-\mathrm{ND}$}

Fábio Marcon Alfieri, Carolyne Freita Vieira, Natália Cristina de Oliveira Vargas e Silva

\section{RESUMO}

Alterações decorrentes do processo de envelhecimento podem impactar o controle postural e a sensibilidade cutânea plantar. Essas mudanças são fatores predisponentes a quedas em idosos. Objetivo: Comparar a sensibilidade cutânea plantar e o controle postural em indivíduos jovens e idosos, e buscar associações entre estas variáveis nestes dois grupos etários. Métodos: Trata-se de um estudo transversal do qual participaram 63 indivíduos de ambos os sexos. Os participantes foram avaliados por meio dos testes Timed Up and Go (TUG), Apoio Unipodal e Estesiometria (por monofilamentos de nylon). Resultados: Os indivíduos jovens exibiram resultados significantemente melhores que os idosos no tempo de execução do TUG, tempo de apoio unipodal e na sensibilidade plantar, avaliada pela estesiometria $(p<0,0001)$. As correlações entres as variáveis do estudo revelaram associações fracas e muito fracas (não significantes), tanto entre os jovens como entre os idosos, exceto pela associação entre tempo de apoio unipodal e estesiometria do lado direito entre os indivíduos jovens $(r=-0,35, p=0,05)$. Conclusões: A sensibilidade plantar e o controle postural são menores em idosos quando comparados com os jovens, e não houve associação entre o teste clínico de controle postural e a sensibilidade plantar avaliada por estesiometria.

PALAVRAS-CHAVE: Postura; Idoso; Adulto; Acidentes por quedas.

\section{ABSTRACT}

Alterations due to the aging process may impact postural control and plantar skin sensitivity. These changes are predisposing factors to falls in the elderly. Objective: To compare plantar skin sensitivity and postural control in young and elderly individuals, and to seek associations between these variables in both age groups. Methods: This is a cross-sectional study involving 63 individuals of both sexes. Participants were evaluated using the Timed Up and Go test (TUG), Unipodal Support and Esthesiometry (with nylon monofilaments). Results: Young individuals presented significantly better results than the elderly at the time of TUG execution, time of unipodal support and plantar sensitivity, evaluated by the esthesiometry $(p<0.0001)$. Correlations between the study variables revealed weak and very weak associations (non-significant), both among the young and the elderly individuals, except for the association between unipodal support time and right-sided esthesiometry among young individuals $(r=-0,35, p=0.05)$. Conclusions: Plantar sensitivity and postural control are lower in the elderly when compared to young individuals, and there was no association between the clinical test of postural control and plantar sensitivity evaluated by esthesiometry.

KEYWORDS: Posture; Aged; Adult; Accidental falls. 


\section{INTRODUÇÃO}

Informações sensoriais advindas dos sistemas vestibular, visual e proprioceptivo contribuem para o controle postural. Dentro deste último sistema, destaca-se a informação da superfície cutânea plantar, que contribui para a percepção espacial e temporal, importantes para a percepção de mudanças de orientação corporal ${ }^{1,2}$.

O processo de envelhecimento envolve importantes alterações fisiológicas. A redução do número de mecanorreceptores localizados nos pés e o aumento do limiar de excitabilidade vibratória nesta região podem levar à redução da capacidade somatossensorial no indivíduo idoso e, portanto, contribuem para a diminuição do controle postura| ${ }^{3,4}$. Além desse fator, limitações visuais, alterações nos sistemas vestibular e musculoesquelético, bem como 0 uso de alguns fármacos podem contribuir para a síndrome geriátrica de instabilidade postural pode levar o idoso à queda 5 .

Sobre o controle postural e a sensibilidade cutânea, avaliados respectivamente pela escala de equilíbrio de Berg e por monofilamentos de nylon, há relato de que, em idosos da comunidade, existe associação entre queixa de perda de equilíbrio e perda de sensibilidade ${ }^{6}$. Ao comparar jovens e idosos por meio de testes visuais, somatossensoriais, motores, e de controle postural, os idosos apresentam desempenho inferior nos testes sensoriais, dentre eles o de sensibilidade cutânea, avaliada por monofilamentos de nylon (Semmes-Weinstein monofilaments), bem como do controle postural avaliado por plataforma computadorizada ${ }^{7}$.

Araneda e Solorza ${ }^{8}$ estudaram a relação entre sensibilidade cutânea plantar e equilíbrio dinâmico em idosos saudáveis, e concluíram que a diminuição da sensibilidade cutânea plantar de ambos os lados teve pequena associação com maiores índices de equilíbrio dinâmico dos idosos.

A diminuição da sensibilidade cutânea (avaliada por monofilamentos de nylon) e do controle postural (avaliado por plataforma de força) também foram observados em idosos quando comparados com indivíduos jovens no estudo de Ueda e Carpes $^{9}$. Os autores verificaram que, em idosos, a amplitude anteroposterior e a área do centro de pressão estiveram associadas com a sensibilidade plantar geral, e, nos jovens, a sensibilidade na região do antepé esteve associada com o melhor controle postural.

A sensibilidade plantar e o controle postural têm funções essenciais para a marcha, e o declínio dessa resposta somatossensorial pode acarretar consequências significativas para os idosos, já que a sensibilidade plantar contribui para o controle da pressão plantar durante a postura ortostática dos idosos ${ }^{10}$. Apesar disso, ainda se discute o quanto a informação advinda da superfície cutânea plantar contribui para a regulação do controle postural em idosos saudáveis.

Dessa forma, compreender a contribuição da sensibilidade plantar para o controle postural, assim como os declínios nessa função sensorial no envelhecimento, podem ajudar no melhor entendimento desta importante questão 
que pode estar relacionada a quedas, já que a diminuição do controle postural é um dos fatores predisponentes a esta condição"11.

Devido à importância da contribuição do sistema proprioceptivo para o controle postural de idosos ${ }^{7}, 0$ objetivo deste estudo foi comparar a sensibilidade cutânea plantar e o controle postural em indivíduos jovens e idosos, e buscar associações entre estas variáveis nestes dois grupos etários.

\section{MÉTODOS}

Trata-se de um estudo transversal observacional. Todos os procedimentos foram aprovados pelo Comitê de Ética em Pesquisa do Centro Universitário Adventista de São Paulo (parecer número 2.117.856), e os participantes deram por escrito sua autorização para participação na pesquisa, através da assinatura de um termo de consentimento livre e esclarecido.

0 recrutamento dos participantes da pesquisa foi feito por meio de contato direto com acompanhantes de pacientes da policlínica universitária de um Centro Universitário privado localizado na zona sul da cidade de São Paulo. Aqueles que preencheram os critérios de inclusão foram chamados para avaliação.

Participaram do estudo 63 indivíduos de ambos os sexos, 31 jovens com idade entre 18 e 30 anos, e 32 pessoas com 60 anos ou mais, capazes de caminhar de maneira independente, sem uso de órteses ou próteses, e que não possuíssem nenhuma lesão nos membros inferiores que pudesse afetar a locomoção ou a postura. A coleta de dados do estudo ocorreu entre os meses de agosto de outubro de 2017.

Não participaram deste estudo aqueles com: déficits neurológicos, compressão de cauda equina, história prévia de trauma em membros inferiores (pés), neuropatias e afeç̧ões nos pés (como por exemplo, neuropatia diabética, bolhas, úlceras ou deformidades), e aqueles com histórico de doenças cerebelares e vestibulares.

Os participantes foram submetidos à uma avaliação composta por: peso, estatura, índice de massa corporal (IMC), mobilidade funcional, e equilíbrio estático e sensibilidade plantar, conforme descrito a seguir.

\section{Avaliação Antropométrica}

Para calcular o IMC foi dividido o peso em quilogramas pelo quadrado da estatura em metros. O IMC foi classificado da seguinte forma: $<20 \mathrm{~kg} / \mathrm{m}^{2}$ - baixo peso; de 20 a $24,99 \mathrm{~kg} / \mathrm{m}^{2}$ - eutrófico; de 25 a 29,99 kg/m² - sobrepeso; $\geq 30 \mathrm{~kg} / \mathrm{m}^{2}$ - obesidade ${ }^{12}$. 


\section{Mobilidade Funcional}

Para mensurar a mobilidade funcional, foi utilizado o teste Timed Up and Go (TUG). $O$ teste consiste em medir em segundos o tempo gasto pelo indivíduo para levantar-se de uma cadeira, andar três metros, voltar e sentar-se novamente na cadeira. $\mathrm{O}$ teste foi repetido três vezes, sendo selecionado para a análise o menor tempo do indivíduo ${ }^{13}$.

\section{Equilíbrio Estático}

Para avaliar o equilíbrio estático foi usado o teste de Apoio Unipodal. O teste consiste em solicitar ao indivíduo que se equilibre em apenas um dos pés com olhos abertos e posteriormente com olhos fechados por no máximo 30 segundos. $O$ tempo que o voluntário permanecer apoiado em somente um dos pés é medido em três tentativas, sendo considerada a melhor delas para a análise. O teste permite identificar alterações de equilíbrio, e aqueles que permanecem em apoio unipodal de olhos abertos por 21 a 30 segundos são classificados como não possuindo alteração do equilíbrio. Neste estudo, o avaliador esteve ao lado do participante durante a realização do teste, a fim de prevenir e evitar quedas ${ }^{14}$.

\section{Sensibilidade Plantar}

A avaliação da sensibilidade plantar foi medida por estesiometria utilizando monofilamentos de nylon (SORRI Bauru, Semmes-Weistein monofilaments), com o mesmo comprimento e diferentes diâmetros, gerando pressão padronizada sobre a pele. A avaliação foi feita conforme a classificação dos monofilamentos e suas cores: verde e azul - sensibilidade normal; violeta - dificuldade de discriminação de forma e temperatura; vermelho - discreta perda da sensação protetora, vulnerável a lesões; laranja - leve perda da sensação protetora; rosa - perda da sensação protetora, nenhuma resposta, perda da sensibilidade total. Um escore numérico foi estipulado para cada cor (verde=1, azul=2, violeta $=3$, vermelho=4, laranja $=5$, e rosa=6). Ambos os pés foram avaliados, nos pontos previamente descritos por Ueda e Carpes9, nas regiões do ante pé, médio pé e retro pé.

As áreas avaliadas foram a superfície digital do hálux, terceiro e quinto dedos, bem como as regiões dos metatarsos respectivos, além do calcâneo e ponto na superfície cutânea da região dorsal (entre $01^{\circ} \mathrm{e} \circ 2^{\circ}$ metatarsos). A somatória dos pontos em cada pé foi utilizada para a análise. 
A análise dos dados foi feita em pacote estatístico Graph Pad Instat. Os dados foram apresentados em médias \pm desvios-padrão. As comparações entre os grupos (jovens e idosos) foram feitas pelo teste t não pareado ou MannWhitney de acordo com a normalidade dos dados (avaliada pelo teste de Kolmogorov-Smirnov). As associações foram calculadas pelo teste de correlação de Pearson ou Spearman, assumindo-se a seguinte classificação: 0 a 0,19 - muito fraca; 0,20 a 0,39 - fraca; 0,40 a 0,59 - moderada; 0,60 a 0,79 - forte; 0,80 a 1,00 - muito forte. Em todos os casos, o nível descritivo a estabelecido foi de $5 \%$.

\section{RESULTADOS}

Participaram deste estudo 63 indivíduos, a maior parte do sexo feminino (88,8\%). Os grupos se mostraram homogêneos em relação à predominância de gênero. Como esperado, houve diferenças significantes entre os grupos no que diz respeito ao IMC, tempo de execução do TUG, tempo de apoio unipodal e escore de estesiometria (Tabela 1). Tabela 1 - Características antropométricas, mobilidade funcional, equilíbrio estático e sensibilidade plantar de jovens e idosos.

\begin{tabular}{|c|c|c|c|}
\hline & Jovens & Idosos & p \\
\hline $\mathbf{n}$ & 31 & 32 & NS \\
\hline Homens/Mulheres & $3 / 28$ & $4 / 28$ & $<0,0001$ \\
\hline Idade (anos) & $20 \pm 1,6$ & $68,6 \pm 7,5$ & 0,02 \\
\hline Peso (kg) & $63,6 \pm 13,8$ & $71,8 \pm 13,6$ & 0,005 \\
\hline Estatura (cm) & $164,5 \pm 8,1$ & $158,4 \pm 8,6$ & $<0,0001$ \\
\hline IMC (kg/m2) & $23,3 \pm 4,3$ & $28,7 \pm 4,5$ & $<0,0001$ \\
\hline TUG (s) & $8,1 \pm 1,2$ & $10,2 \pm 1,6$ & $<0,0001^{*}$ \\
\hline Apoio Unipodal (s) & $29,7 \pm 1,2$ & $20 \pm 10$ & $<0,0001$ \\
\hline Estesiometria D & $16,8 \pm 4,6$ & $26,5 \pm 6,4$ & $<0,0001$ \\
\hline Estesiometria E & $16,1 \pm 3,9$ & $26,2 \pm 8,3$ & \\
\hline
\end{tabular}

IMC: índice de massa corporal; TUG: teste Timed Up and Go; D: lado direito; E: lado esquerdo; kg: quilogramas; cm: centímetros, $\mathrm{Kg} /$ $m^{2}$ : quilogramas por metro quadrado. *teste de Mann-Whitney.

As associações entres as variáveis do estudo revelaram apenas correlações fracas e muito fracas, não significantes, tanto entre os jovens como entre os idosos, exceto pela associação entre tempo de apoio unipodal e estesiometria do lado direito entre os indivíduos jovens $(r=-0,35, p=0,05)$ (Tabela 2). 
Tabela 2 - Correlações entre mobilidade funcional, equilíbrio estático e sensibilidade plantar nos grupos avaliados.

\begin{tabular}{lccccc} 
& \multicolumn{2}{c}{ Jovens } & \multicolumn{2}{c}{ Idosos } \\
\hline & $\mathbf{r}$ & $\mathbf{p}$ & $\mathbf{r}$ & $\mathbf{p}$ \\
\hline TUG vs. Apoio Unipodal & 0,05 & 0,78 & $-0,05$ & 0,76 \\
TUG vs. Estesiometria D & $-0,19$ & 0,28 & 0,24 & 0,20 \\
TUG vs. Estesiometria E & $-0,03$ & 0,86 & 0,27 & 0,15 \\
Apoio Unipodal vs. Estesiometria D & $-0,35$ & 0,05 & 0,06 & 0,75 \\
Apoio Unipodal vs. Estesiometria E & $-0,16$ & 0,37 & 0,06 & 0,76 \\
\hline
\end{tabular}

TUG: teste Timed Up and Go; D: lado direito; E: lado esquerdo.

\section{DISCUSSÃO}

O objetivo deste estudo foi comparar a sensibilidade cutânea plantar e o controle postural em indivíduos jovens e idosos, e buscar associações entre estas variáveis. Indivíduos jovens apresentaram melhores resultados do que indivíduos idosos em relação à mobilidade funcional, equilíbrio e sensibilidade cutânea plantar.

O grupo de jovens exibiu menor peso e consequentemente menor IMC do que o grupo de idosos. Esses dados eram esperados, uma vez que nos idosos ocorre aumento de tecido adiposo ${ }^{15}$.

Em relação à mobilidade funcional (avaliada pelo TUG) observou-se que os indivíduos jovens conseguiram executar o teste em menor tempo, apresentando assim menor risco de quedas. Os indivíduos que realizam o teste em tempo menor que 10 segundos são independentes e não apresentam alterações de equilíbrio ${ }^{16}$. 0 tempo médio de execução do TUG no grupo de idosos ficou muito próximo deste valor, o que evidencia que se tratavam de idosos que provavelmente não possuíam maiores problemas com o equilíbrio dinâmico ${ }^{13}$. Contudo, a diminuição do tempo de teste é desejável, já que uma meta análise mostrou que o tempo de execução do teste em idosos com faixa etária acima dos 60 anos deve ser de, em média, 9,4 segundos ${ }^{17}$.

Situação semelhante foi verificada em relação ao teste de Apoio Unipodal, que avalia o equilíbrio estático. Os idosos deste estudo conseguiram ficar em média 20 segundos apoios em um dos pés, valor este muito próximo da normalidade se considerarmos o tempo de 21 a 30 segundos como valor de referência para este teste ${ }^{18}$.

A sensibilidade cutânea plantar vem sendo estudada com o intuito de avaliar o declínio somatossensorial em idosos ${ }^{4}$. Em relação à estesiometria, que mensura esta sensação, os jovens apresentaram dados significantemente melhores que os idosos, corroborando com os estudos de Ueda e Carpes ${ }^{9}$, Machado et al..$^{19}$ e Toledo et al. ${ }^{7}$. 
Os resultados da sensibilidade plantar e do controle postural (avaliados pelo TUG e Apoio Unipodal) de certa forma eram esperados, pois indivíduos idosos tendem a apresentar diminuição de controle postural e de sensibilidade, devido às alterações no sistema nervoso central e periférico características do processo de envelhecimento, assim como à redução da velocidade de condução nervosa e perda de fibras mielinizadas e não-mielinizadas ${ }^{20}$.

Sobre as associações entre equilíbrio e sensibilidade, não foram observadas correlações significantes no grupo dos idosos. Entre os jovens, houve apenas uma correlação significante, porém fraca, entre o apoio unipodal e estesiometria do lado direito, indicando que quanto pior a sensibilidade, menor o tempo de permanência no teste. Outros estudos ${ }^{6,9}$ encontraram associações entre equilíbrio e sensibilidade, no entanto, esses autores empregaram medidas diretas de avaliação de equilíbrio, como a plataforma de força, associadas à avaliação da sensibilidade por monofilamentos de nylon.

Uma limitação desde estudo reside no fato de o tipo de avaliação da sensibilidade utilizada ter sido uma medida um tanto subjetiva, necessitando de total atenção e compreensão por parte do indivíduo para sua execução. Além disso, a utilização de uma plataforma de equilíbrio para avaliar esta variável poderia também produzir resultados com menos subjetividade. No entanto, optou-se neste estudo por utilizar testes clínicos, que possuem boa aplicabilidade clínica para a avaliação do controle postural em idosos ${ }^{21}$, além de terem fácil acesso e baixo custo para os profissionais da saúde. Futuros estudos que avaliem a força muscular associados à sensibilidade e controle postural também poderão contribuir para o melhor entendimento sobre a relação entre esses fatores, já que publicações sobre esta temática ainda são escassas. Ainda, a avaliação do nível de atividade física e déficit proprioceptivo também podem trazer importantes informações sobre esta questão durante o envelhecimento, e fornecer dados para subsidiar de forma mais assertiva futuros programas de intervenção.

Os resultados deste estudo permitem inferir que o envelhecimento traz alterações na sensibilidade e no controle postural, o que pode levar à implicações clínicas importantes. Indivíduos idosos devem ser incentivados a praticarem exercícios específicos para a melhoria destas capacidades, independentemente da não observância de associações entre estas variáveis neste estudo, que as avaliou por meio de testes clínicos.

\section{CONSIDERAÇÕES FINAIS}

Asensibilidade cutânea plantar de ambos os pés e o controle postural são menores em idosos quando comparados com indivíduos jovens. Não foram estabelecidas associações entre o controle postural e a sensibilidade entre indivíduos idosos e houve uma fraca correlação entre tempo de permanência em apoio unipodal e sensibilidade plantar entre os jovens. 


\section{REFERÊNCIAS}

1. Roll R, Kavoudoudias A, Roll JP. Cutaneous afferents from human plantar sole contribute to body posture awareness. Neuroreport 2002; 13:1957-61.

2. Patel M, Magnusson M, Kristinsdottir E, Fransson PA. The contribution of mechanoreceptive sensation on stability and adaptation in the young and elderly. Eur J Appl Physiol 2009;105(2):167-73.

3. Alfieri FM. Distribuição da Pressão Plantar em idoso após intervenção proprioceptiva. Rev Bras Cineantropom Desempenho Hum 2008;10(2):137-142.

4. Bretan O. Sensibilidade cutânea plantar como risco de queda em idosos. Rev Assoc Med Bras 2012; 58(2):132.

5. Moraes EN, Marino MCA, Santos RR. Principais síndromes geriátricas. Rev Med Minas Gerais 2010;20(1):5466.

6. Bretan O, Pinheiro R M, Corrente J E; Avaliação funcional do equilíbrio e da sensibilidade cutânea plantar de idosos moradores na comunidade. Braz J otorhinolaryngol 2010;76(2):219-224.

7. Toledo DR, Barela JA. Diferenças sensoriais e motoras entre jovens e idosos: contribuição somatossensorial no controle postural. Rev Bras Fisioter 2010; 14(3):267-75.

8. Araneda JE, Solarza EL. Sensibilidad cutánea plantar y balance dinámico en adultos mayores saludables de la comunidad: estudio relacional. Fisioter Pesq 2013;20(4):310-315.

9. Ueda L, Carpes FP. Relação entre sensibilidade plantar e controle postural em jovens e idosos. Rev Bras Cineantropom Desempenho Hum 2013; 215-224.

10. Zhang S, Li L. The differential effects of foot sole sensory on plantar pressure distribution between balance and gait. Gait Posture 2013;37(4): 532-7. 
11. Alfieri FC, Moraes MCL. Envelhecimento e o controle postural. Saúde Coletiva 2008; 4 (19): 30-3.

12. Vannucchi H, Unamuno MRDL, Marchini JS. Avaliação do estado nutricional. Medicina (Ribeirão Preto) 1996; 29:5-18.

13. Podsiadlo D, Richardson S. The Timed Üp\& Go": a test of basic functional mobility for frail elderly persons. J AmGeriatr Soc. 1991; 39:142-148.

14. Gustafson AS, Noaksson L, Kronhed AC, Möller M, Möller C. Changes in balance performance in physically active elderly people aged 73-80. Scand J Rehabil Med 2000;32(4):168-72.

15. Kent-Braun JA, Alexander VNG, Young K. Skeletal muscle contractile and noncontractile components in young and older women and men. J Appl Physiol 2000;88:662-688.

16. Figueiredo KMOB, Lima KC,Guerra RO. Instrumentos de avaliação do equilibrio corporal em idosos. Rev Cineantropom Desempenho Hum 2007; 9(7):408-13.

17. Bohannon RW. Single limb stance times: a descriptive meta-analysis of data from individuals at least 60 years of age. Topics Geriatr Rehabil. 2007; 22(1):70-7.

18. Maciel ACC, Guerra RO. Prevalência e fatores associados ao déficit de equilíbrio em idosos. R. bras. Ci e Mov. 2005;13(1):37-44.

19. Machado AS, Silva CBP, Rocha ES, Carpes FP. Efeitos de uma manipulação da sensibilidade plantar sobre o controle da postura ereta em adultos jovens e idosos. Rev bras reumatol 2017;57(1):30-36.

20. Mold JW, Vesely SK, Keyl BA, Schenk JB, Roberts M. The prevalence, predictors, and consequences of peripheral sensory neuropathy in older adults. J Am Board Fam Pract 2004;17(1):309-18.

21. Alfieri FM, Riberto M, Gatz LS, Ribeiro CP, Battistella LR. Uso de testes clínicos para verificação do controle postural em idosos saudáveis submetidos a programas de exercícios físicos. Acta Fisiatr 2010;17(4):153-158. 